\title{
The dual-task practice advantage: Empirical evidence and cognitive mechanisms
}

\author{
Tilo Strobach ${ }^{1}$
}

Published online: 31 May 2019

(C) The Psychonomic Society, Inc. 2019

\begin{abstract}
Practice of two simultaneous component tasks in dual-task situations leads to an improvement in dual-task performance. The present paper reviews empirical evidence for this practice-related improvement and discusses its underlying cognitive mechanisms. In particular, the robustness of the phenomenon of dual-task practice advantage (DTPA) is evaluated. This phenomenon is described as an advantage in dual-task performance at the end of dual-task practice (i.e., simultaneous task practice) in comparison to the dual-task performance after single-task practice (i.e., separate task practice). The review investigates the existence of empirical evidence of the DTPA phenomenon and specifications of its mechanisms in the context of the allocation and scheduling hypothesis and the integration hypothesis. Evidence for the existence of the DTPA phenomenon and the allocation and scheduling hypothesis was demonstrated in dual tasks with (1) combinations of two sensorimotor tasks, (2) combinations of one continuous task and one sensorimotor task, (3) combinations of two working-memory tasks, and (4) combinations of one motor task and one cognitive task. In contrast, the findings demonstrate that the DTPA phenomenon in dual tasks with (5) combinations of two long-term memory retrieval tasks can instead be explained by the integration hypothesis. The relevance of these findings is discussed in relation to real-world problems.
\end{abstract}

Keywords Dual tasks $\cdot$ Practice $\cdot$ Cognitive mechanisms $\cdot$ Dual-task skills

\section{Introduction}

When performing two tasks simultaneously, real-world experience and data in systematically conducted lab studies demonstrate performance impairments in comparison to separated task performance in single-task situations. These dual-task costs are demonstrated by an increase in reaction times (RTs) and/or error rates under dual tasks in contrast to single tasks. However, numerous studies have shown a strong (and in rare cases even complete) reduction of these costs after dual-task practice. For instance, a practice-related cost reduction was evident in dual-task situations with combinations of sensorimotor tasks (e.g., Göthe, Oberauer, \& Kliegl, 2016; Hazeltine, Teague, \& Ivry, 2002; Kamienkowski, Pashler, Dehaene, \& Sigman, 2011; Ruthruff, Johnston, \& Van Selst, 2001; Ruthruff, Johnston, Van Selst, Whitsell, \& Remington,

Tilo Strobach

tilo.strobach@medicalschool-hamburg.de

1 Department of Psychology, Medical School Hamburg, Am Kaiserkai 1, 20457 Hamburg, Germany
2003; Ruthruff, Van Selst, Johnston, \& Remington, 2006; Van Selst, Ruthruff, \& Johnston, 1999), working-memory (WM) updating tasks (e.g., Oberauer \& Bialkova, 2011; Oberauer \& Kliegl, 2004), memory-retrieval tasks (e.g., Nino \& Rickard, 2003), and motor tasks (e.g., Wollesen \& Voelcker-Rehage, 2013).

Typically, high dual-task costs at the beginning of dual-task practice are explained by interference in one or both component tasks due to capacity limitations in the cognitive system (i.e., processing bottlenecks; Logan \& Gordon, 2001; Meyer \& Kieras, 1997; Pashler, 1994; Townsend \& Wenger, 2004). Generally, it is assumed that this interference is reduced with practice. However, there is debate as to the details of how this interference is reduced and how cognitive mechanisms explain the reduction of dual-task costs with practice. Strobach and Schubert (2017) therefore provided a review of several mechanisms within the domain of dual tasks with simple, well-controlled, and discrete sensorimotor tasks. Most of these simple tasks are fairly straightforward; involve a rather small set of stimuli, responses, and thus stimulus-response mappings; and usually take less than a second to complete. The relative simplicity of these tasks allows for testing 
assumptions regarding precise practice-related mechanisms associated with a reduction of these costs and with improved dual-task performance. However, reviewing practice-related mechanisms within dual tasks only with sensorimotor tasks may limit the validity of the conclusions of Strobach and Schubert to this specific type of dual-task practice. It might be that this dual-task type and its component tasks require different mechanisms for the reduction of dual-task costs than alternative task types and combinations thereof.

A more general contribution of this review is to add to the literature on cognitive plasticity and practice. Theories about underlying cognitive mechanisms that explain practice-related changes are largely lacking and are under-represented in the literature (Strobach \& Karbach, 2016; Taatgen, 2013), and this review aims to provide one step in overcoming this deficit. Such theories should help in gaining knowledge about cognitive practice mechanisms from a scientific point of view, but should also add to solutions of real-world problems (Strobach $\&$ Schubert, 2014). In many such problems, information technology has greatly increased in complexity recently, in domains such as (air) traffic control, unmanned aircraft systems, and laparoscopic surgery. In these domains, operators need to coordinate the performance of multiple tasks with limited attentional resources. Traffic control, for example, incorporates the surveillance of multiple cameras and sensing equipment by a single operator. Although automation tools are being developed and their net effect will likely be to reduce physical workloads, the cognitive workload increases. This increased workload requires optimal performance, which might result from well-designed practice procedures for operators. These practice procedures, however, require knowledge of the underlying cognitive mechanisms in dual-task practice, which is focused on in this review.

\section{The dual-task practice advantage and its mechanisms}

To pursue the aim of looking for mechanisms of improved dual-task performance with practice, the present paper evaluates the robustness of one specific phenomenon: the dual-task practice advantage (DTPA), an idea going back to the empirical findings of Hirst, Spelke, Reaves, Caharack, and Neisser (1980) as well as to the theoretical assumptions of Damos and Wickens (1980): "[single-task skills] fail, in short, to account for the development of timesharing skills that may be unique to the multiple-task situation, such as the parallel processing of information, rapid switching between tasks, or the use of efficient response strategies" (p. 16). The DTPA phenomenon can thus be described as an advantage in dual-task performance at the end of dual-task practice in comparison with the dual-task performance after single-task practice, i.e., when component tasks are practiced in separated conditions.
To generate an overarching theoretical contribution, the present review considers two general explanations of cognitive mechanisms underlying the DTPA phenomenon: the allocation and scheduling hypothesis and the integration hypothesis. According to the former, dual-task practice induces participants to adopt a strategy of coordinating the two tasks, which enables them to develop dual-task skills of cognitive resource allocation and scheduling. For instance, such resource allocation and scheduling is generally proposed by the executive-process interactive control theory. In the context of this theory, Meyer and Kieras (1997) developed an adaptive executive control model to account for dual-task costs. This model assumes that according to specific task demands, the processing stages of two component tasks can be performed either in parallel or sequentially when strategic bottlenecks are introduced. In contrast, alternative structural bottleneck models assume unavoidable bottlenecks at processing stages with mentally demanding, capacity-limited operations (e.g., stimulus-response mapping stages in sensorimotor tasks; Pashler, 1994; Pashler \& Johnston, 1998). However, both strategic and structural models suggest that controlling bottlenecks requires allocation and scheduling processes (e.g., Jiang, Saxe, \& Kanwisher, 2004; Lien, Schweickert, \& Proctor, 2003; Szameitat, Lepsien, von Cramon, Sterr, \& Schubert, 2006; Szameitat, Schubert, Müller, \& Von Cramon, 2002); for instance, these extensions assume switching processes for inhibition, activation, and instantiation of task information between component tasks and their bottleneck stages (Band \& van Nes, 2006; Lien et al., 2003).

Irrespective of the strategic or structural nature of bottlenecks, the first general prediction of the allocation and scheduling hypothesis is that dual-task practice enables development of the improved dual-task skills of cognitive resource allocation and scheduling (Kieras, Meyer, Ballas, \& Lauber, 2000; Oberauer \& Kliegl, 2004; Schumacher et al., 2001); the same prediction can be derived from a theory like that proposed by Gopher (1993), who argued that sharing attention between two tasks is a skill that can be achieved with dual-task practice. One characteristic of such dual-task skills in the context of the allocation and scheduling hypothesis is that this hypothesis does not exclude a generalization of these skills beyond practiced tasks. Hence, these skills could be at least partially independent from the tasks presented during practice. If so, the DTPA phenomenon is transferable to alternative unpracticed dual tasks or other task situations. This assumption of transferable dual-task skills acquired during practice is consistent with the general assumption of transferable cognitive control and executive processes (e.g., Karbach \& Kray, 2016).

Alternatively, according to the integration hypothesis, dualtask practice might produce an efficient integration of two tasks and combine them, in an extreme case, into a single super task (e.g., Hazeltine, Aparicio, Weinstein, \& Ivry, 
2007; Hazeltine et al., 2002; Oberauer \& Kliegl, 2004; Ruthruff et al., 2006). Whereas two separate tasks and their separate capacity-limited processes are performed at the beginning of practice, a single capacity-limited process of the combined task is processed after dual-task practice. The processing of two capacity-limited processes increases the likelihood of dual-task costs (e.g., due to sequential processing of task components), while the likelihood is reduced with only one such process. However, this mechanism proposed in the context of the integration hypothesis should exclusively shape the processing of the two tasks combined during practice since integration exclusively works for task information processed during practice. So, in contrast to the allocation and scheduling hypothesis, the integration hypothesis predicts that the DTPA phenomenon is specific to practiced tasks, is not independent from these tasks, and cannot be transferred to alternative task situations after practice.

Irrespective of the general mechanism explaining the DTPA phenomenon (i.e., the allocation and scheduling hypothesis versus the integration hypothesis), this phenomenon describes mechanisms beyond mechanisms of single-task practice with separate practice of component tasks. Such mechanisms of single-task practice are related to the concept of automatization (e.g., Fisk \& Schneider, 1984; Logan \& Gordon, 2001; Schneider \& Shiffrin, 1977) and the shortening of processing stages in tasks (e.g., Pashler \& Baylis, 1991) that are combined in dual-task situations (e.g., Dux et al., 2009; Kamienkowski et al., 2011; Maquestiaux, LaguëBeauvais, Bherer, \& Ruthruff, 2008; Ruthruff et al., 2006; Strobach, Liepelt, Pashler, Frensch, \& Schubert, 2013; Van Selst et al., 1999). Evidence for these mechanisms (i.e., automatization and shortening of component tasks) comes from studies that demonstrated reduced dual-task costs after separate practice of two tasks in single-task situations. In contrast to dual-task practice, however, processes of allocation and scheduling are not required in single-task situations. So such processes are not practiced and no improved dual-task skills are acquired. Similarly, single-task practice should not lead to an integration of processes of two component tasks because these tasks are not processed simultaneously during this practice type.

\section{The present review}

As a consequence of introducing the potential effects of dualtask practice, the explicit aims of this paper are: (1) Is there empirical evidence for the DTPA phenomenon? If so, does this evidence exist across dual-task types that differ with regard to the combined component tasks? (2) If the DTPA phenomenon exists across different dual-task practice types, what are the specific cognitive changes that can explain this phenomenon (i.e., practice mechanisms)? Is there evidence demonstrating transfer effects beyond the practiced dual-task situation to inform about the allocation and scheduling hypothesis versus the integration hypothesis and the mechanisms associated with these hypotheses? These questions will guide the remainder of this paper, across different types of dual tasks and thus different combinations of component tasks: (1) combinations of two sensorimotor tasks, (2) combinations of one continuous task and one sensorimotor task, (3) combinations of two WM tasks, (4) combinations of one motor task and one cognitive task, and (5) combinations of two long-term memory tasks (a literature overview is illustrated in Table 1).

\section{Dual-task combinations of two sensorimotor tasks}

\section{Empirical evidence}

Empirical findings demonstrating that the DTPA phenomenon is no trivial phenomenon come from a study by Ruthruff et al. (2006). In that study, the authors investigated the potential existence of this phenomenon in a dual-task situation of the psychological refractory period (PRP) type. In this dual-task type, a first Task 1 (e.g., an auditory sensorimotor task) and a second Task 2 (e.g., a visual sensorimotor task) are separated by a varying time interval (i.e., stimulus onset asynchrony [SOA]), and participants are instructed to respond as fast and as accurately as possible with priority on Task 1 . The applied component tasks included a relatively high number of stimulus-response mappings: four plus eight stimulusresponse mapping rules in Task 1 and Task 2, respectively, and these mappings were demanding due to a mix of compatible and incompatible mappings between stimulus and response information. Both the number and the compatibility relation of the stimulus-response mappings resulted in highly complex tasks in Ruthruff et al. (2006). Dual-task costs in PRP dual-task situations are generally indicated by increasing RTs in Task 2 with decreasing SOA. This RT increase is the prominent PRP effect (Pashler, 1994; Welford, 1952) and is explained by a structural (e.g., Pashler, 1994; Welford, 1952, 1980) or a strategic limitation (e.g., Hazeltine, Ruthruff, \& Remington, 2006; Meyer \& Kieras, 1997; Miller, Ulrich, \& Rolke, 2009) in processing two stimulus-response mappings at the same time (Fig. 1). Practice-related reductions of the PRP effect would indicate dual-task improvement.

The existence of the DTPA in the PRP context was investigated by Ruthruff et al. (2006) in three practice groups. While Group 1 practiced Task 1 and Task 2 under PRP dualtask conditions for eight sessions, Group 2 practiced only Task 1 , and Group 3 practiced only Task 2 for the same amount of time under single-task conditions. In the final test sessions, all three groups performed the same dual-task situation that Group 1 performed during practice. One of the main findings 
Table 1. Overview of types of dual tasks and their studies investigating the phenomenon of dual-task practice advantage (DTPA). According to the author's knowledge, all studies with a test of dual-task performance after dual-task practice and single-task practice were incorporated within the dual-task types and no study with such a test was excluded

\begin{tabular}{ll}
\hline Type of dual tasks with ... & Studies investigating the DTPA phenomenon \\
\hline$\ldots$ two sensorimotor tasks & - Liepelt, Strobach, Frensch, \& Schubert (2011) \\
& - Ruthruff, Van Selst, Johnston, \& Remington (2006) \\
& - Schubert \& Strobach (2018) \\
& - Schubert, Liepelt, Kübler, \& Strobach (2017) \\
& - Strobach, Frensch, Müller, \& Schubert (2015) \\
& - Strobach, Frensch, Soutschek, \& Schubert (2012) \\
$\ldots$ one continuous task and one sensorimotor task & - Strobach, Salminen, Karbach, \& Schubert (2014a) \\
$\ldots$ two working-memory tasks & - Anguera et al. (2013) \\
... one motor task and one cognitive task & - Bender, Filmer, Naughtin, \& Dux (2017) \\
& - Oberauer \& Kliegl (2004) \\
& - Salminen, Kühn, Frensch, \& Schubert (2016) \\
... two long-term memory tasks & - Perumal, Melam, Alhusaini, Buragadda, \& Sharma (2017) \\
& - Silsupadol, Siu, Shumway-Cook, \& Woollacott (2006) \\
& - Silsupadol et al. (2009) \\
\hline
\end{tabular}

of the test sessions was the observation of a similar PRP effect in the dual-task Group 1 and the single-task Group 2 that practiced only Task 1 (Ruthruff et al., 2006; Group 3 showed still an increased PRP effect). This finding shows no

\section{a Dual-task group}

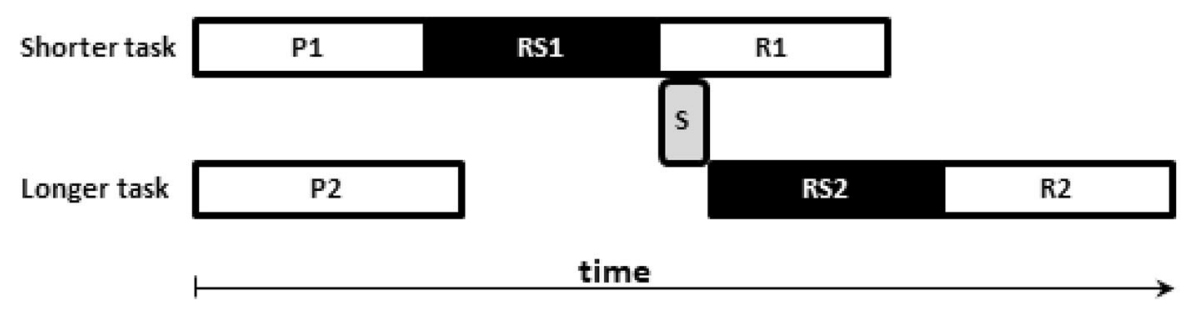

\section{b Single-task group}

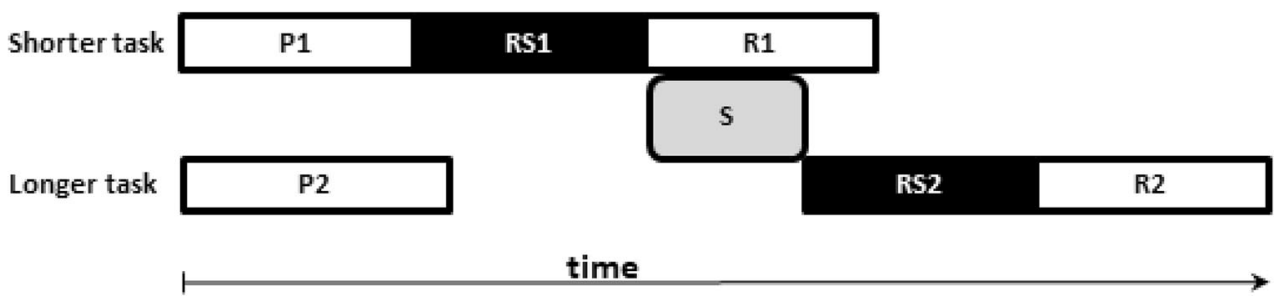

Fig. 1. Dual-task processing architecture after dual-task practice (Panel A: Dual-task group) and single-task practice (Panel B: Single-task group) with two sensorimotor tasks. According to bottleneck models, central response-selection (RS) stages in sensorimotor tasks are processed sequentially even with practice (Maquestiaux, Hartley, \& Bertsch, 2004; Ruthruff et al., 2003) while perception (P1, P2) and response (R1, R2) stages are processed in parallel. A potential switching stage (S) after the RS stage in the shorter task (RS1) and before this stage in the longer task (RS2) is shortened after dual-task practice in contrast to single-task practice (Schubert \& Strobach, 2018). This phenomenon is a promising candidate to explain improved dual-task performance after experience with dual tasks in contrast to this performance after single-task experience (the dual-task practice advantage). Note that the latencies of the individual processing stages (i.e., P1, P2, RS1, RS2, S, R1, R2) in the figure are schematic illustrations and may not represent actual stage latencies of a combination of tasks with a first response (i.e., the visual task) and a second response (i.e., the auditory task) (Liepelt et al., 2011; Strobach et al., 2015; Strobach et al., 2012; Strobach et al., 2014a) 
existence of the DTPA phenomenon and is consistent with the assumption that the practice-related improvement of dual-task performance is not related to this phenomenon when combining highly complex sensorimotor tasks.

The first empirical evidence in favor of the DTPA phenomenon was provided by Liepelt, Strobach, Frensch, and Schubert (2011) as well as Strobach, Frensch, Soutschek, and Schubert (2012), with combinations of less complex sensorimotor tasks. In their studies, a visual-manual task (i.e., visual task) and an auditory-verbal task (i.e., auditory task) were presented in separate single-task trials and were presented simultaneously in dual-task trials $(\mathrm{SOA}=0 \mathrm{~ms}$; Hazeltine et al., 2002; Schumacher et al., 2001); both component tasks included sets of three stimulus-response pairs and compatible mappings between stimulus and response information. The dual-task costs were investigated as the performance impairment in dual tasks in comparison to single tasks. While dual-task practice included presentations of the visual and auditory tasks in single-task and in dualtask blocks, single-task practice included the presentation of both tasks in single-task blocks exclusively. In fact, after six practice sessions in the dual-task group, dual-task performance was improved, with reduced dual-task costs in the seventh test session when compared to the dual-task performance after six sessions of single-task practice; single-task performance did not differ. In detail, the dual-task cost reduction was exclusively demonstrated by reduced RT costs in the auditory task, while there was no such evidence in the visual task (Liepelt et al., 2011; Strobach et al., 2012). A comparison of the specific magnitudes of the costs in the auditory task RTs after dual-task practice and single-task practice shows that the dual-task groups produced only $25 \%$ and $28 \%$ of the costs of the single-task groups in Liepelt et al. (2011; Experiment 1) and Strobach et al. (2012; Experiment 1), respectively. Since the auditory task is typically executed after the visual task (i.e., the auditory task is performed after the visual task; see also Hartley, Maquestiaux, \& Butts, 2011; Schumacher et al., 2001; Strobach, Frensch, \& Schubert, 2008; Tombu \& Jolicœur, 2004), the DTPA is associated with the secondly performed component task in this dual-task situation.

Evidence for the existence of the DTPA phenomenon in the studies of Liepelt et al. (2011) and Strobach et al. (2012) was demonstrated in young adults (aged between 19 and 29 years). However, Strobach, Frensch, Müller, and Schubert (2015) showed that this phenomenon in dual tasks with a combination of two sensorimotor tasks is age-independent to some degree. Dual-task practice in comparison to single-task practice in older adults (aged between 57 and 68 years) also resulted in reduced dual-task costs in the RTs of the longer auditory component task; a comparison of the specific magnitudes of the costs shows that the dual-task group produced only $43 \%$ of the costs in the single-task group.

\section{Practice mechanisms}

What are the practice-related mechanisms that might explain the DTPA phenomenon in dual tasks with two less complex sensorimotor tasks? To answer this question, it is important to look for evidence of transfer beyond the practiced tasks. If there is transfer, this finding would support the allocation and scheduling hypothesis, while no evidence of transfer is consistent with the assumptions of the integration hypothesis. Importantly, findings showed improved dual-task performance in dual-task groups in comparison to single-task groups when the identical components are presented during practice and during the dual-task test at the end of practice (as shown in Experiment 1 of Liepelt et al., 2011; Strobach et al., 2015; Strobach et al., 2012). However, this DTPA phenomenon was also evident when stimuli and stimulus-response mappings of the visual and/or the auditory task were changed in the final dual-task tests (Liepelt et al., 2011, Experiments 2 \& 3; Schubert, Liepelt, Kübler, \& Strobach, 2017; Strobach et al., 2015); thus, the DTPA phenomenon was independent of the specific perceptual and stimulus-response mapping processes in these tasks. These findings are not consistent with the assumption that both tasks were integrated into one supertask representation, which leaves only one integrated bottleneck (e.g., at the response selection mechanism).

This transfer of the DTPA phenomenon to changed dual tasks, however, is predicted by the allocation and scheduling hypothesis and its assumption of acquired and improved dualtask skills (Hirst et al., 1980; Kramer, Larish, \& Strayer, 1995). As illustrated in Fig. 1, one specific realization of these skills that explains the DTPA phenomenon in the longer auditory task might be as follows: The dual-task processing architecture includes (1) a capacity limitation (i.e., a bottleneck process) in the shorter visual task (e.g., at a central response selection stage) followed by (2) a switching operation (related to the inhibition of mapping rules of irrelevant tasks as well as activation and instantiation of such rules in relevant tasks such as the longer auditory task in WM), and (3) the capacity limitation in the longer auditory task (Band \& van Nes, 2006; Lien et al., 2003). After dual-task practice (Fig. 1A) in contrast to single-task practice (Fig. 1B), inhibition, activation, and instantiation processes are highly efficient, leading to a shortening of the switching operation and improved dual-task performance in the longer auditory task (Strobach, Salminen, Karbach, \& Schubert, 2014a, for a more detailed discussion). A theoretically important aspect is that the DTPA phenomenon might be limited to less complex tasks, but cannot be generalized to highly complex tasks (Ruthruff et al., 2006; Schubert \& Strobach, 2018). Less complex tasks put a rather low load on WM capacity, which enables participants to acquire and apply dual-task skills, while highly complex tasks with a high load on WM capacity enable no such skill acquisition and application (Schubert \& Strobach, 2018). 


\section{Dual-task combinations of two working memory (WM) tasks}

\section{Empirical evidence}

An early study investigating the DTPA phenomenon in the context of dual tasks with two WM tasks was published by Oberauer and Kliegl (2004). In this study, young participants practiced updating two items in WM through two sequences of operations (one numerical operation: a high tone requiring the addition of 2 and a low tone subtraction of 1 from the current value of a digit; one spatial operation: spatial operations were indicated by visually presented red arrows, displayed in the central cell of a grid and pointing in one of eight possible directions horizontally, vertically, or diagonally; these arrows required the mental shift of a dot from its current position in the indicated direction by one cell). In different blocks, stimuli for the two sequences of operations were presented either separately in alternating order or simultaneously. At the beginning of the dual-task practice, participants experienced substantial dual-task costs which might result from the capacity-limited nature of the WM component as well as the processes to coordinate different sets of information in this component. However, at the end of six to 12 practice sessions, operation latencies for simultaneous processing were similar to the latencies for the two operations in the alternating singletask condition, suggesting improved dual-task performance. Important for the present context, this dual-task improvement increased the improvement after separate single-task practice of the numerical and spatial operations (Oberauer \& Kliegl, 2004), demonstrating the DTPA phenomenon with two WM tasks. A comparison of the specific magnitudes of the dualtask costs in these tasks after dual-task practice and single-task practice (Oberauer \& Kliegl, 2004) shows that the dual-task group produced only about $14 \%$ of the costs of the single-task group.

Such a demonstration was also evident in a study combining two $n$-back WM tasks (Salminen, Kühn, Frensch, \& Schubert, 2016); in such $n$-back tasks, sequences of stimuli are presented and participants have to decide for each sequence whether the current stimulus matches the one that was presented $n$ stimuli back in the sequence. In detail, two groups of participants practiced with $n$-back tasks, including visual position and auditory letter stimuli, for 16 days (Jaeggi, Buschkuehl, Jonides, \& Perrig, 2008; see Soveri, Antfolk, Karlsson, Salo, \& Laine, 2017, for a recent meta-analysis). While the dual-task group practiced both tasks simultaneously, there was isolated practice of the visual and the auditory $n$ back tasks in the single-task group. In both groups, the $n$-back situations were designed in an adaptive way so that, when participants responded correctly in most of the trials (e.g., 18 correct responses in 20 trials), the level of $n$ increased by one (e.g., participants were advanced from 2-back to 3-back). In contrast, it was decreased by one (e.g., from 3-back to 2-back), if participants performed a lower number of correct responses (e.g., less than 15 correct responses in 20 trials), while the $n$ back level remained constant in all other cases. As a consequence, the achieved $n$-back level under the dual-task condition is a valid indicator of practiced dual-task performance in the $n$-back task.

To test transfer, a WM updating task with sequences of items was presented and the length of the sequences varied randomly. Participants were unaware of the length of the ongoing sequence and were asked to constantly update the contents of their WM. Under single-task conditions, sequences included either auditory numbers or visual bars at different locations, and participants had to report the last four numbers or bar locations, respectively. Under dual-task conditions, sequences of auditory and visual items were presented simultaneously and participants were asked to report either the auditory items or the visual items; item modality was unpredictable before the end of the sequences. The WM updating task can be considered a transfer task since the practiced $n$-back and the updating task differed with respect to stimuli (squares and letters in the $n$-back tasks versus digits and stimulus locations in the updating tasks) and task rules; the $n$-back task requires the recognition of previously presented items while the updating task requires recollection and reproduction of item information.

When comparing the achieved $n$-back level in the practice task at the end of practice in the dual-task and single-task group, there was improved dual $n$-back performance in the former group while single $n$-back performance did not differ (Salminen et al., 2016); a comparison of the specific magnitudes of the dual-task costs in the achieved $n$-back level after dual-task practice and single-task practice shows that the dualtask group actually produced a dual-task benefit of $96 \%$ of these costs in the single-task group (Table 1). Thus, this study validated the DTPA phenomenon in dual tasks with two WM tasks. This validation was not limited to the practiced $n$-back task, but was also evident in the WM updating transfer tasks. While the dual-task group showed improved performance under the dual-task updating condition at the end of practice (i.e., the number of correctly reported items), there was no such improvement in the single-task conditions in comparison to the single-task group.

\section{Practice mechanisms}

Oberauer and Kliegl (2004) assumed that the DTPA phenomenon could be explained by learning to combine two WM tasks. However, their design was not able to validate this hypothesis in contrast to the allocation and scheduling hypothesis because the authors conducted no transfer conditions. Such conditions are required to test for the acquisition of dual-task skills in the context of this latter hypothesis. 
Accordingly, Salminen et al. (2016) strongly focused on the potential transfer effects to non-practiced tasks after dual-task practice with two simultaneous WM tasks. The analysis of these transfer effects might show which non-practiced tasks benefit from practice and might offer a window onto the shared processing components between practiced and nonpracticed tasks; such a benefit might also inform about the cognitive changes, explaining the DTPA phenomenon. In fact, the practice of Salminen et al. produced evidence for transfer: The DTPA transferred to the dual-task condition of the nontrained WM updating task, but not to single-task versions of this updating task and the $n$-back single tasks; the single-task group showed no such transfer to the non-practiced dual-WM updating task. Thus, dual $n$-back practice improved skills that are not specific to the practiced task situation but are transferable. These transferable skills explain the DTPA phenomenon in dual $n$-back tasks in the context of the allocation and scheduling hypothesis, while these skills are inconsistent with the integration hypothesis that does not predict any transfer beyond the practice tasks. These acquired skills optimize processes generalizable to all types of "WM updating of stimuli from two modalities" (Salminen et al., 2016, p. 10198); such skills are not required when only one stimulus is processed during single $n$-back practice. In the context of multicomponent WM models, such generalizable skills could be conceptualized in an executive control module that coordinates updating of stimulus processing in different short-term memory components. For the exemplary case of Baddeley's (2012) WM model, a central executive applies these skills on a phonological loop (to update the auditory letter information) and on a visuo-spatial sketch pad (to process the visual position information).

\section{Dual-task combinations of one motor task and one cognitive task}

\section{Empirical evidence}

Silsupadol et al. (2009) investigated both the impairment of and the effects of practice on the control of motor balance under dual-task conditions in older adults. Because impaired dual-task balance performance predicts adverse outcomes like falling as well as declines in both cognitive and physical functioning, interventions that improve dual-task balance performance are crucial and inform about real-world problems. During the dual-task practice of this study, different balance tasks, such as standing with eyes closed, walking backward, and catching a ball, were performed simultaneously with cognitive tasks (e.g., naming objects or remembering numbers), while the balance tasks were practiced separately during single-task practice. After practice, dual-task performance on balance was tested in combination with a set of other cognitive tasks, such as addition/subtraction questions. In this balance test after practice, participants had to walk $10 \mathrm{~m}$ and the time to complete the middle $6 \mathrm{~m}$ (i.e., gait speed in $\mathrm{m} / \mathrm{sec}$ ) in combination with the cognitive tasks was measured. Dual-task costs in the balance task at low practice levels might be explained by interference between this task and the cognitive tasks because of capacity-limited attentional requirements of locomotor and cognitive control (Woollacott \& ShumwayCook, 2002).

In essence, dual-task gait speed was increased after dualtask practice in comparison to single-task practice effects during the balance test (Silsupadol et al., 2009). Such an increase was not evident under single-task conditions, reflecting the DTPA phenomenon; a comparison of the specific magnitudes of the dual-task costs in gait speed after dual-task practice and single-task practice shows that the dual-task practice condition produced only $81 \%$ of the costs of single-task practice conditions. These findings replicate and extend the findings of a prior single case study (Silsupadol, Siu, Shumway-Cook, \& Woollacott, 2006). However, a recent study was not able to replicate the DTPA phenomenon in patients with Parkinson's disease (Perumal, Melam, Alhusaini, Buragadda, \& Sharma, 2017). The results of this study showed that dual-task practice is not more effective than single-task practice in improving dual-task balance performance of these patients. Thus, similar to dual tasks with two sensorimotor tasks (Ruthruff et al., 2006), the DTPA phenomenon is no trivial finding and is not generalizable to all conditions. One might speculate that patients with Parkinson's disease do not possess the potential capacity and plasticity as non-patients do to produce a DTPA.

\section{Practice mechanisms}

What is the specific cognitive change that explains faster gait speed in dual tasks after dual-task practice (i.e., the DTPA phenomenon)? Silsupadol et al. (2009) assumed that their findings support the assumption of the development of dualtask skills for the optimized attentional control of balance and cognitive tasks during dual-task practice. This assumption is based on the finding that the tasks during balance practice and balance test differed (e.g., balance tasks: standing with eyes closed, catching a ball vs. walking/cognitive tasks: naming objects vs. addition questions). So the DTPA phenomenon was evident in an unpracticed transfer task at the final test. These findings show that potentially acquired dual-task skills are not tied to the specific practice tasks, but are transferable between dual-task situations with different balance tasks. These conclusions are consistent with the allocation and scheduling hypothesis when explaining the DTPA phenomenon in the context of combinations of a motor task and a cognitive task. In contrast, these conclusions are not consistent with the integration hypothesis, which does not predict such transfer between different situations. 


\section{Dual-task combinations of one continuous task and one sensorimotor task}

\section{Empirical evidence}

Dual tasks combining one continuous task and one sensorimotor task were realized in situations with a video race game and a detection task, respectively (Anguera et al., 2013). The participants with the video race game were instructed to track a car on a simulated road with left/right turns as well as uphill/ downhill sections, while the participants of the detection task were instructed to execute a response when a green sign, among red signs, was presented. Older adults in a dual-task group and a single-task group practiced both tasks either simultaneously or in separate single-task blocks, respectively, across 12 sessions and 4 weeks. Dual-task costs in the detection task (i.e., signal-detection performance under single tasks in comparison to dual tasks) were substantial in both the dualtask and the single-task group at the beginning of practice and vaguely explained by interference between tasks. These costs were, however, reduced in the dual-task group in contrast to the single-task group at the end of practice (Anguera et al., 2013); a comparison of the specific magnitudes of the dualtask costs after dual-task practice and single-task practice shows that the dual-task group produced only $37 \%$ of the costs in the single-task group. This study thus realized the DTPA phenomenon with one continuous task and one sensorimotor task, and the phenomenon was even evident in a follow-up after 6 months.

Bender, Filmer, Naughtin, and Dux (2017) extended the findings of Anguera et al. (2013) to a group of young and middle-aged participants and a new set of continuous and sensorimotor tasks. That is, across six sessions of practice, a dual-task and a single-task practice group performed a visuomotor-tracking task and a shape-discrimination task simultaneously and separately, respectively. In the visuomotortracking task, participants had to continuously pursue a visually presented moving black target with a computer mouse so that a black cursor remained as close as possible to the center of the moving target, while those in the discrimination task were instructed to detect a colored shape target among distractor stimuli. The data across these component tasks demonstrated similar dual-task costs in the single-task group and the dual-task group before practice. After practice, these costs were mainly reduced in the discrimination task in the dualtask group compared to the single-task group (the former group produced only about $55 \%$ of the costs in the latter group), illustrating the DTPA phenomenon.

\section{Practice mechanisms}

To investigate the specific cognitive changes that might explain the DTPA phenomenon in dual tasks with a continuous and a sensorimotor task, the study by Anguera et al. (2013) provides the opportunity to analyze the effects beyond the practiced task situation. In such non-practiced transfer situations, the dual-task group, in comparison to the single-task group, showed significantly improved performance in the domains of WM and sustained attention; the former domain is investigated by a delayed-recognition task, while the latter is measured by more rapid responses in a vigilance test. However, these transfer improvements were limited to WM and sustained attention processes, and were not the result of generalized increases in speed of processing, as the dual-task practice group of Anguera et al. did not improve in two tests of processing speed. Further, there was no significant improvement in a letter-number dual-task test (multitasking with two sensorimotor tasks), in the useful field of view test (visual attention), as well as in a change-detection task (visual shortterm memory). The lack of improvement is consistent with the transfer investigations of Bender et al. (2017) in a nonpracticed PRP dual task that showed no better dual-task performance in the dual-task group in contrast to the single-task group. These authors were also not able to show transfer effects on an attentional blink test, Stroop task, Flanker task, or Go-No-go task (investigating inhibition and action control).

Importantly, however, the transfer effects to WM and sustained attention (Anguera et al., 2013) suggest that a common, underlying mechanism of cognitive coordination was challenged and enhanced by dual tasks with one continuous and one sensorimotor task; however, this common mechanism is not fully general and does not boost performance in alternative domains, for example visual attention. Focused on the aim to specify practice-related mechanisms explaining the DTPA phenomenon, these findings thus suggest that practice with simultaneous continuous and sensorimotor tasks improves dual-task skills that are not specific for the practice situation, but this practice improves skills that are transferable to some extent (at least to processes of WM and sustained attention). These conclusions on the transferability of these skills are consistent with the allocation and scheduling hypothesis and are inconsistent with assumptions of the integration hypothesis.

\section{Dual-task combinations of two long-term memory tasks}

\section{Empirical evidence}

The investigation of the DTPA phenomenon and its transfer effects in the context with two long-term memory tasks has a crucial difference from the previously reported dual-task combinations in that dual-task practice and single-task practice were not realized in different groups of participants. In this case, dual-task and single-task practice were realized in the 
same of group of participants, but in different sets of cues on which long-term memory practice was performed (Orscheschek, Strobach, Schubert, \& Rickard, 2019; Strobach, Schubert, Pashler, \& Rickard, 2014b). ${ }^{1}$ In detail, participants had to associate each of a set of 14 color-word cues with a unique spatial key-press or a unique vocal-digit response in the beginning of practice on the long-term memory tasks. After forming these associations, participants were given multiple triads of blocks of retrieval practice. Within each triad, there was one single-retrieval key-press block, one single-retrieval vocal-digit block, and one dual-retrieval block; dual-retrieval blocks required participants to execute both the key-press and the vocal-digit responses. Importantly, one set of seven cues (old cues) received practice under single- and dual-retrieval conditions, while the other set of seven cues was practiced under single-retrieval conditions exclusively (new cues).

At the beginning of practice, RTs of the first and second responses on old cues in dual-retrieval blocks (i.e., RT1 and RT2, respectively) were above the single-retrieval RTs in the vocal-digit and key-press blocks. Dual-retrieval RTs above single-retrieval RTs could be explained by a bottleneck at the memory retrieval stage of processing and only sequential retrieval of information on two responses from long-term memory. During practice, however, a subgroup of participants showed an RT pattern on old cues with short inter-response intervals (IRIs) between RT1 and RT2, and these participants gave very reduced RT2s at the end of three practice sessions, indicative of improved dual-retrieval performance. Since RT2s violated the lower bound prediction of a mathematical sequential retrieval model, some form of learned parallelism was acquired by this subgroup of participants. In a transfer phase following this learned retrieval parallelism at the end of the practice, old and new cues were presented intermixed in the same single- and dual-retrieval blocks. RT2 on old cues still demonstrated learned retrieval parallelism, while RT2 on new cues did not, which was consistent with a sequential retrieval assumption; single-task RTs of old and new cues did not show a difference during the transfer phase (Strobach, Schubert, et al., 2014). A comparison of the specific magnitudes of the dual-task costs after dual-retrieval practice shows that participants actually produced a dualtask benefit of about $224 \%$ on old cues in comparison to the costs on new cues. Thus, dual tasks with two long-term memory retrieval tasks showed the DTPA phenomenon on old cues with dual-retrieval practice in comparison to new cues without such practice.

\footnotetext{
${ }^{1}$ Nino and Rickard (2003) investigated the effects of single-retrieval practice and dual-retrieval practice. Although they produced findings consistent with the assumption of the DTPA phenomenon, they realized these types of practice in different experiments and different designs that do not warrant a systematic comparison of both practice types and their effects.
}

\section{Practice mechanisms}

The findings of the dual-retrieval practice studies (Orscheschek et al., 2019; Strobach, Schubert, et al., 2014) are incompatible with assumptions of the allocation and scheduling hypothesis since there is no evidence for transfer of learned retrieval parallelism between old and new cues within the same blocks of dual-memory retrieval (i.e., old and new cues were presented under very similar conditions). Alternatively, the literature on dual tasks with two long-term memory tasks discusses one variation of the integration hypothesis. According to this discussion, participants with reduced IRIs learned to chunk the two responses independently for each cue, such that both responses could be retrieved in one pass through a potential retrieval bottleneck that appears to govern performance at the beginning of the practice (Nino \& Rickard, 2003). That chunking process may only be possible when both responses are concurrently in WM, as should be the case for old cues and low IRIs on their responses. Because response chunking is an associative memory process that would occur during a dual-retrieval trial, this response chunking account suggests that learned retrieval parallelism occurs at the individual cue-response level (i.e., for old cues only) rather than at the task level (i.e., for old and new cues). Consistent with that possibility, Orscheschek et al. (2019) and Strobach, Schubert, et al. (2014) have shown that this parallelism did not transfer to cues for which only single-retrieval practice occurred during the practice phase, with dual-retrieval trials introduced only during a transfer phase. In this way, empirical evidence in dual tasks with two long-term memory retrieval tasks showed the existence of the DTPA phenomenon and provided evidence that is consistent with the integration hypothesis (i.e., the chunking mechanism; Nino \& Rickard, 2003). ${ }^{2}$

\section{Conclusions}

The aims of the present paper were to evaluate the robustness of the DTPA phenomenon, assuming an advantage in dualtask performance at the end of dual-task practice in comparison with the dual-task performance after single-task practice,

\footnotetext{
${ }^{2}$ Note that the absence of an empirical generalization to other cues is consistent with the integration hypothesis. Although this absence is not consistent with the assumptions of the allocation and scheduling hypothesis, it does not conclusively allow a differentiation between this hypothesis and the integration hypothesis, because it might be that learning in the context of the allocation and scheduling hypothesis is (for some reason) specific to the practiced long-term memory tasks or that the selection of another set of tests would produce such a transfer. However, it is assumed that this latter argument is rather unlikely in the present context, since if this would be the case then the specificity would have been highly focused and skills would not generalize to cues within the same set of cues, presented in the same blocks, and with the same instructions.
} 
as well as to specify mechanisms explaining this phenomenon. As a first approach to specify practice-related mechanisms in an overarching theoretical framework, findings consistent with the allocation and scheduling hypothesis versus the integration hypothesis were looked for. The DTPA phenomenon in dual tasks was investigated with (1) combinations of two sensorimotor tasks, (2) combinations of one continuous and one sensorimotor task, (3) combinations of two WM tasks, (4) combinations of one motor and one cognitive task, and (5) combinations of two long-term memory tasks.

First, all considered types of dual tasks and age groups provided empirical evidence consistent with the existence of the DTPA phenomenon. Thus, this phenomenon is robust across dual-task types and is age-independent to some degree, since the DTPA occurred in young and older adults. So empirical evidence for the DTPA phenomenon is generally consistent with the theoretical assumptions of Damos and Wickens (1980). The specific magnitudes of the DTPA phenomenon differ tremendously from dual-task benefits after dual-task practice (i.e., improved dual-task performance in comparison to single-task performance after dual-task practice, while single-task practice produced dual-task costs as in dual-task combinations of two WM tasks) to only small DTPAs (i.e., slightly reduced dual-task costs after dual-task practice in comparison to these costs after single-task practice as in dual-task combinations of one motor task and one cognitive task).

Second, the review of potential mechanisms explaining the DTPA phenomenon generally showed empirical evidence consistent with the acquisition of dual-task skills in the context of the allocation and scheduling hypothesis in dual tasks with (1) combinations of two sensorimotor tasks, (2) combinations of one continuous task and one sensorimotor task, (3) combinations of two WM tasks, and (4) combinations of one motor task and one cognitive task. However, the specifications of these skills across the different types of dual-task practice are as diverse as the number of these situations at present, that is, there is low generalizability of the specific mechanisms explaining dual-task skills in the context of the allocation and scheduling hypothesis (e.g., combinations of two sensorimotor tasks: skills for a faster switch between central response-selection stages; combinations of two WM tasks: improved updating of stimuli from two modalities in WM).

These conclusions differ partly from those of studies combining two long-term memory tasks. Such studies also produced evidence consistent with the assumption of the DTPA phenomenon. However, they offer no evidence that is consistent with the acquisition of dual-task skills (Strobach, Schubert, et al., 2014). Rather, findings in studies with two long-term memory tasks supported the assumption of chunking of two memory retrievals, thus the integration and restructuring of the architecture of the long-term memory tasks. So the integration hypothesis provides a promising practice-related mechanism in the context of dual tasks with two long-term memory tasks.

Differences between the consequences of the different dual-task practice types reviewed in this paper call for future studies that include similar empirical tests of their effects. Such studies could, for instance, investigate the consequences of the different practice types in the same set of transfer tasks. These transfer tasks should be designed in a way in which they allow for an elaborated analysis of the underlying cognitive mechanisms that explain the DTPA phenomenon across different practice types, thus allowing for a specification of the allocation and scheduling hypothesis.

Referring to real-world problems, the present review can provide some suggestions. First, although the realworld situations with multiple tasks might differ from the ones investigated in the reviewed studies, it might be that also real-world dual-task situations and practice in these situations could show the DTPA phenomenon. From the author's perspective, studies on situations with one continuous task and one sensorimotor task (Anguera et al., 2013; Bender et al., 2017) represent a first step from lab research towards modeling real-world dual-task situations. Second, if the DTPA phenomenon also exists in real-world problems, this suggests practicing these problems' multiple tasks in dual tasks, but not in isolation as in single-task practice. Third, investigating practice combinations with two long-term memory tasks produced no evidence for transferable dual-task skills (i.e., this practice type provided evidence for the integration hypothesis), while findings in studies with other combinations were consistent with the allocation and scheduling hypothesis. That is, practicing these task combinations leads to the acquisition of improved dual-task skills that are not specific for the practice situations, but are at least partially independent of these situations and are transferable. Although the findings on the characteristics of transfer are diverse across the different types of dual-task practice, it is plausible that a dualtask practice is transferable to and between real-world problems. From a broader perspective, the present review on the DTPA phenomenon adds to the general literature on cognitive plasticity and practice. That is, this review specifies theoretical assumptions about the underlying cognitive mechanisms in the context of the allocation and scheduling hypothesis as well as the integration hypothesis that explain changes after dual-task practice in contrast to single-task practice.

Acknowledgements The present paper was supported by a Research Grant of the German Research Foundation (Deutsche Forschungsgemeinschaft, STR 1223/1-1). Correspondence concerning this article should be addressed to Tilo Strobach, Medical School Hamburg, Department of Psychology, Am Kaiserkai 1, 20457 Hamburg, Germany. Emails may be sent to tilo.strobach@medicalschool-hamburg.de. 


\section{References}

Anguera, J. A., Boccanfuso, J., Rintoul, J. L., Al-Hashimi, O., Faraji, F., Janowich, J., . . . Johnston, E. (2013). Video game training enhances cognitive control in older adults. Nature, 501(7465), 97-101.

Baddeley, A. (2012). Working memory: theories, models, and controversies. Annual Review of Psychology, 63, 1-29.

Band, G. P., \& van Nes, F. T. (2006). Reconfiguration and the bottleneck: Does task switching affect the refractory period effect? European Journal of Cognitive Psychology, 18(4), 593-623.

Bender, A. D., Filmer, H. L., Naughtin, C. K., \& Dux, P. E. (2017). Dynamic, continuous multitasking training leads to task-specific improvements but does not transfer across action selection tasks. npj Science of Learning, 2: 14.

Damos, D. L., \& Wickens, C. D. (1980). The identification and transfer of timesharing skills. Acta Psychologica, 46(1), 15-39.

Dux, P. E., Tombu, M. N., Harrison, S., Rogers, B. P., Tong, F., \& Marois, R. (2009). Training improves multitasking performance by increasing the speed of information processing in human prefrontal cortex. Neuron, 63(1), 127-138.

Fisk, A. D., \& Schneider, W. (1984). Memory as a function of attention, level of processing, and automatization. Journal of Experimental Psychology: Learning, Memory, and Cognition, 10(2), 181-197.

Gopher, D. (1993). The skill of attention control: Acquisition and execution of attention strategies. In D. E. Meyer \& S. Kornblum (Eds.), Attention and performance XIV: Synergies in experimental psychology, artificial intelligence, and cognitive neuroscience (pp. 299322). Cambridge, MA: MIT Press.

Göthe, K., Oberauer, K., \& Kliegl, R. (2016). Eliminating dual-task costs by minimizing crosstalk between tasks: The role of modality and feature pairings. Cognition, 150, 92-108.

Hartley, A. A., Maquestiaux, F., \& Butts, N. S. (2011). A demonstration of dual-task performance without interference in some older adults. Psychology and Aging, 26(1), 181-187.

Hazeltine, E., Aparicio, P., Weinstein, A., \& Ivry, R. B. (2007). Configural response learning: The acquisition of a nonpredictive motor skill. Journal of Experimental Psychology: Human Perception and Performance, 33(6), 1451-1467.

Hazeltine, E., Ruthruff, E., \& Remington, R. W. (2006). The role of input and output modality pairings in dual-task performance: Evidence for content-dependent central interference. Cognitive Psychology, 52(4), 291-345.

Hazeltine, E., Teague, D., \& Ivry, R. B. (2002). Simultaneous dual-task performance reveals parallel response selection after practice. Journal of Experimental Psychology: Human Perception and Performance, 28(3), 527.

Hirst, W., Spelke, E. S., Reaves, C. C., Caharack, G., \& Neisser, U. (1980). Dividing attention without alternation or automaticity. Journal of Experimental Psychology: General, 109(1), 98.

Jaeggi, S. M., Buschkuehl, M., Jonides, J., \& Perrig, W. J. (2008). Improving fluid intelligence with training on working memory. Proceedings of the National Academy of Sciences, 105(19), 68296833.

Jiang, Y., Saxe, R., \& Kanwisher, N. (2004). Functional magnetic resonance imaging provides new constraints on theories of the psychological refractory period. Psychological Science, 15(6), 390-396.

Kamienkowski, J. E., Pashler, H., Dehaene, S., \& Sigman, M. (2011). Effects of practice on task architecture: Combined evidence from interference experiments and random-walk models of decision making. Cognition, 119(1), 81-95.

Karbach, J., \& Kray, J. (2016). Executive functions. In T. Strobach \& J. Karbach (Eds.), Cognitive training - An overview of features and applications (pp. 93-103). New York, NY: Springer.

Kieras, D. E., Meyer, D. E., Ballas, J. A., \& Lauber, E. J. (2000). Modern computational perspectives on executive mental processes and cognitive control: Where to from here. In S. Monsell \& J. Driver (Eds.), Control of cognitive processes: Attention and performance XVIII (pp. 681-712). Cambridge, MA: MIT Press.

Kramer, A. F., Larish, J. F., \& Strayer, D. L. (1995). Training for attentional control in dual task settings: a comparison of young and old adults. Journal of Experimental Psychology: Applied, 1(1), 50-76.

Lien, M.-C., Schweickert, R., \& Proctor, R. W. (2003). Task switching and response correspondence in the psychological refractory period paradigm. Journal of Experimental Psychology: Human Perception and Performance, 29(3), 692-712.

Liepelt, R., Strobach, T., Frensch, P., \& Schubert, T. (2011). Improved intertask coordination after extensive dual-task practice. The Quarterly Journal of Experimental Psychology, 64(7), 1251-1272.

Logan, G. D., \& Gordon, R. D. (2001). Executive control of visual attention in dual-task situations. Psychological Review, 108(2), 393.

Maquestiaux, F., Hartley, A. A., \& Bertsch, J. (2004). Can practice overcome age-related differences in the psychological refractory period effect? Psychology and Aging, 19(4), 649-667.

Maquestiaux, F., Laguë-Beauvais, M., Bherer, L., \& Ruthruff, E. (2008). Bypassing the central bottleneck after single-task practice in the psychological refractory period paradigm: Evidence for task automatization and greedy resource recruitment. Memory \& Cognition, 36(7), 1262-1282.

Meyer, D. E., \& Kieras, D. E. (1997). A computational theory of executive cognitive processes and multiple-task performance: Part 2. Accounts of psychological refractory-period phenomena. Psychological Review, 104(4), 749-791.

Miller, J., Ulrich, R., \& Rolke, B. (2009). On the optimality of serial and parallel processing in the psychological refractory period paradigm: Effects of the distribution of stimulus onset asynchronies. Cognitive Psychology, 58(3), 273-310.

Nino, R. S., \& Rickard, T. C. (2003). Practice effects on two memory retrievals from a single cue. Journal of Experimental Psychology: Learning, Memory, and Cognition, 29(3), 373.

Oberauer, K., \& Bialkova, S. (2011). Serial and parallel processes in working memory after practice. Journal of Experimental Psychology: Human Perception and Performance, 37(2), 606-614.

Oberauer, K., \& Kliegl, R. (2004). Simultaneous cognitive operations in working memory after dual-task practice. Journal of Experimental Psychology: Human Perception and Performance, 30(4), 689-707.

Orscheschek, F., Strobach, T., Schubert, T., \& Rickard, T. (2019). Two retrievals from a single cue: A bottleneck persists across episodic and semantic memory. Quarterly Journal of Experimental Psychology, 72(5), 1005-1028.

Pashler, H. (1994). Dual-task interference in simple tasks: Data and theory. Psychological Bulletin, 116(2), 220-244.

Pashler, H., \& Baylis, G. (1991). Procedural learning: I. Locus of practice effects in speeded choice tasks. Journal of Experimental Psychology: Learning, Memory and Cognition, 17, 20-32.

Pashler, H., \& Johnston, J. C. (1998). Attentional limitations in dual-task performance. In H. Pashler (Ed.), Attention (pp. 155-189). Hove, England: Psychology Press/Erlbaum (Uk) Taylor \& Francis.

Perumal, V., Melam, G. R., Alhusaini, A. A., Buragadda, S., \& Sharma, N. (2017). Instruction prioritization in task-based balance training for individuals with idiopathic Parkinson's disease. Somatosensory \& Motor Research, 34(1), 27-33.

Ruthruff, E., Johnston, J. C., \& Van Selst, M. (2001). Why practice reduces dual-task interference. Journal of Experimental Psychology: Human Perception and Performance, 27(1), 3.

Ruthruff, E., Johnston, J. C., Van Selst, M., Whitsell, S., \& Remington, R. (2003). Vanishing dual-task interference after practice: Has the bottleneck been eliminated or is it merely latent? Journal of Experimental Psychology: Human Perception and Performance, 29(2), $280-289$

Ruthruff, E., Van Selst, M., Johnston, J. C., \& Remington, R. (2006). How does practice reduce dual-task interference: Integration, 
automatization, or just stage-shortening? Psychological Research, $70(2), 125-142$

Salminen, T., Kühn, S., Frensch, P. A., \& Schubert, T. (2016). Transfer after Dual n-Back Training Depends on Striatal Activation Change. Journal of Neuroscience, 36(39), 10198-10213.

Schneider, W., \& Shiffrin, R. M. (1977). Controlled and automatic human information processing: I. Detection, search, and attention. Psychological Review, 84(1), 1-57.

Schubert, T., Liepelt, R., Kübler, S., \& Strobach, T. (2017). Transferability of dual-task coordination skills after practice with changing component tasks. Frontiers in Psychology, 8:956.

Schubert, T., \& Strobach, T. (2018). Practice-related optimization of dualtask performance: Efficient task instantiation during overlapping task processing. Journal of Experimental Psychology: Human Perception and Performance, 44(12), 1884-1904.

Schumacher, E. H., Seymour, T. L., Glass, J. M., Fencsik, D. E., Lauber, E. J., Kieras, D. E., \& Meyer, D. E. (2001). Virtually perfect time sharing in dual-task performance: Uncorking the central cognitive bottleneck. Psychological Science, 12(2), 101-108.

Silsupadol, P., Shumway-Cook, A., Lugade, V., van Donkelaar, P., Chou, L.-S., Mayr, U., \& Woollacott, M. H. (2009). Effects of single-task versus dual-task training on balance performance in older adults: a double-blind, randomized controlled trial. Archives of Physical Medicine and Rehabilitation, 90(3), 381-387.

Silsupadol, P., Siu, K.-C., Shumway-Cook, A., \& Woollacott, M. H. (2006). Training of balance under single-and dual-task conditions in older adults with balance impairment. Physical Therapy, 86(2), 269-281.

Soveri, A., Antfolk, J., Karlsson, L., Salo, B., \& Laine, M. (2017). Working memory training revisited: A multi-level meta-analysis of n-back training studies. Psychonomic Bulletin \& Review, 24(4), 1077-1096

Strobach, T., Frensch, P., Müller, H., \& Schubert, T. (2015). Evidence for the acquisition of dual-task coordination skills in older adults. Acta Psychologica, 160, 104-116.

Strobach, T., Frensch, P., \& Schubert, T. (2008). The temporal stability of skilled dual-task performance. Paper presented at the Cognitive Science 2007. Proceedings of the 8th Annual Conference of the Cognitive Science Society of Germany. Saarbrücken.

Strobach, T., Frensch, P., Soutschek, A., \& Schubert, T. (2012). Investigation on the improvement and transfer of dual-task coordination skills. Psychological Research, 76(6), 794-811.

Strobach, T., \& Karbach, J. (2016). Cognitive training. New York, NY: Springer.

Strobach, T., Liepelt, R., Pashler, H., Frensch, P., \& Schubert, T. (2013). Effects of extensive dual-task practice on processing stages in simultaneous choice tasks. Attention, Perception, \& Psychophysics, 75(5), 900-920.

Strobach, T., Salminen, T., Karbach, J., \& Schubert, T. (2014a). Practicerelated optimization and transfer of executive functions: a general review and a specific realization of their mechanisms in dual tasks. Psychological Research, 78(6), 836-851.

Strobach, T., \& Schubert, T. (2014). Positive consequences of actionvideo game experience on human cognition: Potential benefits on a societal level. In K. K. Mak (Ed.), Epidemiology of online game addiction.: OMICS Group.

Strobach, T., \& Schubert, T. (2017). Mechanisms of practice-related reductions of dual-task interference with simple tasks: data and theory. Advances in Cognitive Psychology, 13(1), 28-41.

Strobach, T., Schubert, T., Pashler, H., \& Rickard, T. (2014b). The specificity of learned parallelism in dual-memory retrieval. Memory \& Cognition, 42(4), 552-569.

Szameitat, A. J., Lepsien, J., von Cramon, D. Y., Sterr, A., \& Schubert, T. (2006). Task-order coordination in dual-task performance and the lateral prefrontal cortex: an event-related fMRI study. Psychological Research, 70(6), 541-552.

Szameitat, A. J., Schubert, T., Müller, K. U., \& Von Cramon, D. Y. (2002). Localization of executive functions in dual-task performance with fMRI. Journal of Cognitive Neuroscience, 14(8), 1184-1199.

Taatgen, N. A. (2013). The nature and transfer of cognitive skills. Psychological Review, 120(3), 439-471.

Tombu, M., \& Jolicœur, P. (2004). Virtually no evidence for virtually perfect time-sharing. Journal of Experimental Psychology: Human Perception and Performance, 30(5), 795-810.

Townsend, J. T., \& Wenger, M. J. (2004). A theory of interactive parallel processing: new capacity measures and predictions for a response time inequality series. Psychological Review, 111(4), 1003-1035.

Van Selst, M., Ruthruff, E., \& Johnston, J. C. (1999). Can practice eliminate the Psychological Refractory Period effect? Journal of Experimental Psychology: Human Perception and Performance, 25(5), 1268-1283.

Welford, A. T. (1952). The 'psychological refractory period'and the timing of high-speed performance - a review and a theory. British Journal of Psychology. General Section, 43(1), 2-19.

Welford, A. T. (1980). The single-channel hypothesis. In A. T. Welford (Ed.), Reaction times (pp. 215-252). London: Academic Press.

Wollesen, B., \& Voelcker-Rehage, C. (2013). Training effects on motorcognitive dual-task performance in older adults. European Review of Aging and Physical Activity, 11:122.

Woollacott, M., \& Shumway-Cook, A. (2002). Attention and the control of posture and gait: a review of an emerging area of research. Gait \& Posture, 16(1), 1-14.

Publisher's note Springer Nature remains neutral with regard to jurisdictional claims in published maps and institutional affiliations. 